\title{
The Spatial Distribution of Ices in Star-Forming Regions
}

\author{
K. M. Pontoppidan ${ }^{1}$ E. F. van Dishoeck ${ }^{1}$, E. Dartois ${ }^{2}$, H. J. Fraser ${ }^{3}$, \\ Z. Banhidi ${ }^{4}$, J. K. Jørgensen ${ }^{5}$ and the ${\text { c2d } \text { Team }^{6}}^{6}$ \\ ${ }^{1}$ Leiden Observatory, P.O.Box 9513, NL-2300 RA Leiden, Netherlands \\ email: pontoppi@strw.leidenuniv.nl \\ ${ }^{2}$ IAS-CNRS, Bat 121, Université Paris Sud, 91405 Orsay Cedex, France \\ ${ }^{3}$ Department of Physics, University of Strathclyde, 107 Rottenrow, Glasgow G4 0NG, Scotland \\ ${ }^{4}$ Stockholm Observatory, AlbaNova University Center, SE-106 91 Stockholm, Sweden \\ ${ }^{5}$ Harvard-Smithsonian Center for Astrophysics, 60 Garden Street, MS42, Cambridge, MA \\ 02138, USA \\ ${ }^{6}$ http://peggysue.as.utexas.edu/SIRTF/
}

\begin{abstract}
We present results from an ongoing program to map the spatial distribution of ices in dark cloud cores with the Spitzer Space Telescope and VLT-ISAAC. The ice maps are used to directly trace the freeze-out of $\mathrm{CO}$ and the formation of $\mathrm{H}_{2} \mathrm{O}, \mathrm{CH}_{3} \mathrm{OH}$ and $\mathrm{CO}_{2}$.
\end{abstract}

Keywords. infrared: ISM — ISM: abundances — dust, extinction — molecular processes

\section{Introduction}

A significant fraction of the molecular component in dense clouds exists in the form of condensed ices. In the densest star-forming cores, more than half of all molecules apart from $\mathrm{H}_{2}$ can be frozen out onto dust grains. Thus, a full understanding of the chemical state of a molecular cloud requires detailed observations of the structure and abundances of common ices such as water, $\mathrm{CO}_{2}, \mathrm{CH}_{3} \mathrm{OH}, \mathrm{NH}_{3}$ and $\mathrm{CO}$. The only way to directly observe ice in dense interstellar clouds is via absorption bands in the mid-infrared wavelength regime. Thus, infrared continuum sources located behind or inside the cloud of interest are required. The sensitivity of ground-based 8-10 m class telescopes and the Spitzer Space Telescope now allows for spectroscopy of fainter sources concentrated close enough in the plane of the sky to produce multiple lines of sight through the same cloud fragment. Combining multiple lines of sight will produce a spatial map of the abundances of solid state species in a given cloud.

\section{Observations and Results}

Several observing programs using both the Very Large Telescope and Spitzer to map the spatial variation of the abundances of different ice species in a variety of dark clouds have recently been completed. The maps are obtained by selecting cloud regions with a high density of bright background stars or low-luminosity, highly extincted T Tauri stars. Absorption spectra of the ices present along the line of sight are obtained with both ground- and space-based mid-infrared spectrometers. Combining the spectra from different lines of sight produces a map of ices with spatial resolutions of 10-60", comparable to maps of gas-phase molecules obtained with single-dish millimeter telescopes.

These results include a Spitzer $5-40 \mu \mathrm{m}$ ice map of the protostellar envelope of the class 0 protostar Serpens SMM 4 (see also Pontoppidan et al. 2004). Additionally, we 


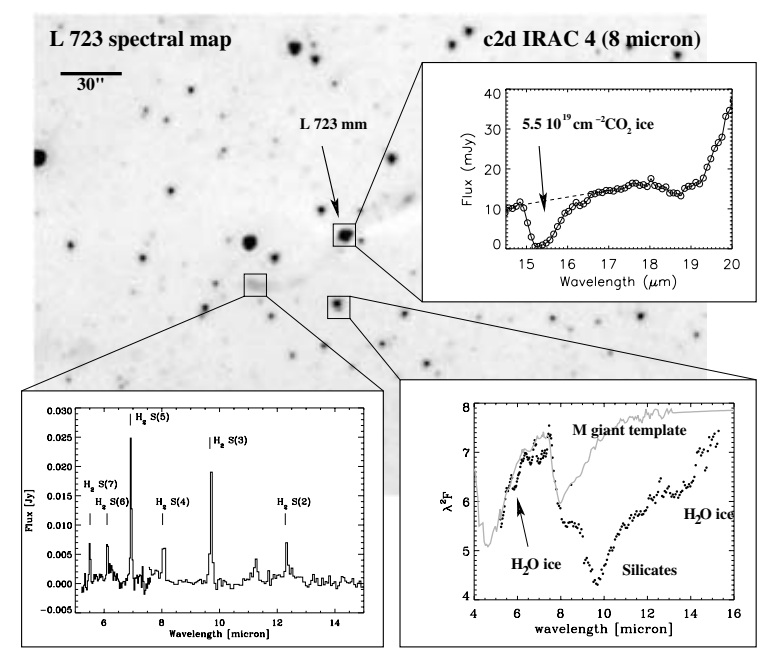

Figure 1. Examples of the Spitzer low-resolution spectra obtained for an ice map of the isolated core L723. Most of the data are spectra of background M and K giants to probe the solid state species in the cloud. We have detected a very high column density of $\mathrm{CO}_{2}$ ice toward the central class 0 object (Dartois et al. 2005, A\&A, in press).

have obtained a Spitzer map of water and $\mathrm{CO}_{2}$ ice of the isolated core L723 as well as a combined $\mathrm{CO} / \mathrm{CO}_{2}$ ice map of the OphF core in the Ophiuchus molecular complex (Pontoppidan et al., in prep.) obtained using ISAAC on the VLT and Spitzer-IRS.

We generally find that the local abundance of water ice is almost constant at $5-9 \times 10^{-5}$ w.r.t. $\mathrm{H}_{2}$ in most dark cloud environments, and only increases beyond this at very high densities. The abundance of CO ice is found to be highly density dependent in cold cloud environments in accordance with simple freeze-out models and CO gas-phase observations (Jørgensen et al. 2005 and references therein). In the Oph F core, the CO ice abundance can be traced down to a freeze-out fraction of $5 \%$ using ground-based spectroscopy of the $4.67 \mu \mathrm{m} \mathrm{C}-\mathrm{O}$ stretching mode. The abundance of $\mathrm{CO}_{2}$ ice is moderately density dependent and increases in abundance by a factor of two for the Oph $\mathrm{F}$ core from the outer part at $50000 \mathrm{AU}$ to the innermost $5000 \mathrm{AU}$. The profile of the $\mathrm{CO}_{2}$ bending mode indicates that the $\mathrm{CO}_{2}$ ice formed at higher densities is very dilute in the $\mathrm{CO}$ ice that dominates the ice mantle during heavy depletion. Direct comparison between the CO stretching and $\mathrm{CO}_{2}$ bending mode profiles shows that the ratio of $\mathrm{CO}$ to $\mathrm{CO}_{2}$ molecules during heavy depletion is $\sim 40$. We suggest that this is a direct measure of the formation rate of $\mathrm{CO}_{2}$ relative to the freeze-out rate of $\mathrm{CO}$ at densities higher than a few $10^{5} \mathrm{~cm}^{-3}$.

\section{Acknowledgements}

Astrochemistry in Leiden is supported by a Spinoza grant from the Netherlands Organization of Scientific Research (NWO). This work is based in part on observations made with the Spitzer Space Telescope (GO-3336 and the Legacy Science program, under contract 1224608), which is operated by the Jet Propulsion Laboratory, California Institute of Technology under NASA contract 1407.

\section{References}

Pontoppidan, K. M., van Dishoeck, E. F., \& Dartois, E. 2004, A\&A 426, 925

Jørgensen, J. K., Schöier, F. L., \& van Dishoeck, E. F. 2005, A\&A 435, 177 